\title{
THE MAYA VENUS NUMBERS
}

\section{Lynn E. Rose}

State University of New York at Buffalo

Cognitive Science Center and Department of Philosophy

\begin{abstract}
Resumen: La tabla de Venus del Códice de Dresde utiliza los misteriosos "números de Venus" 236, 90,250 y 8 . Generalmente se considera que estos números se refieren a la visibilidad como el Lucero del Alba, la invisibilidad de la conjunción superior, la visibilidad como el Lucero de la Tarde, e invisibilidad de la conjunción inferior. Lo que quiere decir que son muy imprecisos. Después de considerar algunos de los intentos recientes para darle sentido a estos números, propongo un enfoque muy distinto: tomo los 90 dias como una ventana dentro de la cual ocurre la invisibilidad de la conjunción superior, y los ocho dias como una ventana en la cual ocurre el ascenso heliaco del Lucero del Alba. Los 236 y 250 se vuelven simplemente los intervalos entre estas dos ventanas. Sostengo que los números mayas de Venus probablemente eran exactos al dia. El resto del artículo se dedica a la investigación de las latitudes y de los valores del arcus visionis que mejor encajan con estos números de Venus.
\end{abstract}

Aвsтract: The Venus table of the Dresden Codex uses the mysterious "Venus numbers": 236, 90, 250 , and 8. Usually these numbers are taken as referring to the visibility as Morning Star, the invisibility at superior conjunction, the visibility as Evening Star, and the invisibility at inferior conjunction. This means that they are highly unrealistic. After considering some of the recent attempts to make sense of these numbers, I propose a quite different approach: 1 take 90 days as a window within which the superior conjunction invisibility occurs, and the $\mathbf{8}$ days as a window within which the heliacal rising of the Morning Star occurs. Numbers 236 and 250 are then simply the intervals between the two windows. I argue that Maya Venus numbers were probably accurate to the day. The remainder of the article is devoted to an investigation of the latitudes and arcus visionis values that would best fit these Venus numbers.

At the lower left of pages 46-50, the Codex Dresden contains the numbers $236,90,250$, and 8 . These numbers are prominently featured as the intervals in the so-called "Venus table" that is continued across the left-hand columns of each of those five consecutive pages. (They are also featured on the Codex Grolier - albeit incompletely, since so much less of the Venus material has survived on the Grolier.)

\section{The standard view}

Nearly always, it has just been assumed that the 236 , the 90 , the 250 , and the 8 are, respectively: the period of the visibility of Venus as the Morning Star; the period of the invisibility of Venus at superior conjunction; the period of the visibility of Venus as the Evening Star; and the period of the invisibility of Venus at inferior 
conjunction. The Codex Dresden says none of this, however, and simply gives us the four Venus numbers. The exact significance of those four numbers is left unspecified. ${ }^{1}$

Notice that $236+90+250+8=584$. In the scholarly literature, this 584-day period is usually called a Venus Round, or "VR" for short. "VR" is much better than the sometimes-favored "VP" - for Venus Period. As the Maya well knew, 584 days is actually a little more than the mean synodic period of Venus! (The modern figure is just under 583.916 days.)

But why use those four numbers? Surely the Maya would have been able to discern that the Evening Star did not routinely shine for 14 days longer than the Morning Star. (Our own experience indicates that during a single synodic period either one of those two visibilities might indeed surpass the other by about that much, but that over longer and longer periods of time their averages will tend to be almost equal.)

Surely the Maya would also have been able to detect that the longer invisibility never lasted as long as 90 days, and that the shorter invisibility might last anywhere from a few days to two or three weeks, depending upon the circumstances.

Yet we are asked to believe that the Maya continued to use their grossly inaccurate figures, through one synodic period after another! If they were so preoccupied with Venus, why is it that they were never able to get their Venus numbers right? Had they no grasp at all of what they were doing? ${ }^{2}$

\section{The Babylonian observations of Venus}

Let us consider for a moment the much-debated Babylonian observations of Venus, or "Ninsianna". It has long been recognized that the intervals found in one portion of the Ninsianna document bear a puzzling resemblance to the Maya Venus numbers; this mysterious similarity should be kept in mind as we proceed.

The surviving fragments of the Ninsianna document date from about twenty-seven hundred years ago. They have frequently been called the Venus Tablets of Ammisaduqa - though it is now being recognized by increasing numbers of scholars that these tablets probably have nothing at all to do with King Ammisaduqa of the First Babylonian Dynasty!

The Ninsianna document is usually divided into Sections I, II, III, and IV, plus the terminal colophon. There is also a Corrigenda Section on many exemplars; it is always placed just before Section IV, or just before the colophon if there is no Section IV on that exemplar.

Section II of the Ninsianna document is often called the Artificial Insertion. This Artificial Insertion is clearly an idealization of some kind, for it consistently recognizes invisibilities of exactly 90 days and of exactly 7 days, which are themselves always separated from each other by visibilities of exactly 8 months 5 days, or 245 days. (The months on the Ninsianna document are always of exactly 30 days.)

The obvious question is: Are the 90 days and the 7 days of the Ninsianna document somehow

\footnotetext{
1 Sometimes a putative translation of the Codex Dresden will attempt such specification. Thus the "translation" given by Anthony Aveni, on page 98 of his Conversing With the Planets, merely supplies the phrases "having been seen" and "having been absent". (Each phrase is supplied twice.) But it needs to be emphasized that there is no such language in the text itself.

${ }^{2}$ See J. Eric S. Thompson, "Maya Astronomy", in Hodson, The Place of Astronomy in the Ancient World, p. 97: "One must try and get in the skin of the Maya priest-astronomer." That will also be our obligation here, at least with respect to these four Venus numbers employed by the Maya. (Since the Maya priest-astronomers sometimes wore the skins of victims that they flayed, this talk of now getting into their skins has a rather bizarre twist to it! But perhaps turn-about is fair play!)
}

Estudios de Cultura Maya. Vol. XXI, 2000 
related to the 90 days and the 8 days of the Maya?

At first glance, any cultural diffusion would seem rather unlikely, since the Ninsianna fragments are from Babylonia, on the other side of the world, and since they are from so much earlier than the conventional "fluorescence" of the Maya - by a millennium or more. (Or could the ancestors of the so-called "Classic" Maya have been the ones involved here?)

\section{How it was done}

Raymond C. Vaughan and I have argued that the "Venus numbers" of the Artificial Insertion (245, 90,245 , and 7) had in fact been derived from the apparently-empirical data of Section I. This derivation would have been accomplished in an arbitrary way, and even in an inappropriate way, but it does nonetheless seem to have been done. $^{3}$

The observations in Section I can be characterized as follows:

The average of the eastern visibilities is $\mathbf{2 4 0 . 2}$ days.

If we include the two longer inferior conjunction invisibilities of 9 months 4 days and of 1 month 7 days, then the average of the superior conjunction invisibilities is 90 days, exactly. (This way of taking the average might have seemed justified because all of the invisibilities that were longer than, say, one month were now being grouped together.)

The average of the longer western visibilities (excluding a rather surprising visibility of only 7 days) is 249.44 days.

The median of the shorter inferior conjunction invisibilities is 7 days. (With regard to the use of the median here, see pages $42-43$ of our paper. Also, remember that the two longer inferior conjunction invisibilities were already averaged in with the superior conjunction invisibilities.)

Finally, it should be noted that the average of the 240.2 and the 249.44 is 244.82 . This would of course round off to 245 days, or 8 months 5 days - which is the very interval that is employed throughout Section II!

Given that Section II uses 90 and 7, and given as of just now that Section I has its longer western visibilities some 9 days longer than its eastern visibilities, and given that the Codex Dresden uses 90 and 8 , with the intervening "western" period 14 days longer than the "eastern" period, we are again faced with that same intriguing question: could there have been some kind of Mesopotamian-Mesoamerican influence - in either direction? (Note also that the western figure that we derived from Section I is 249.44 , which in terms of actual observation would presumably have to be raised to 250 - the very same as the "western" figure that we find in the Codex Dresden. $)^{4}$

Vaughan and I continue to believe that the Section II figures were derived from the Section I material. Thus we do not believe that the Babylonians got their Section II from Mesoamerica (unless, of course, they also got their Section I from Mesoamerica!). But could the Maya or their predecessors have gotten something like Section II from the Babylonians? Perhaps, but it seems rather unlikely. In any case, the Venus materials of these two widely-separated peoples do not establish any such influence - in either direction.

${ }^{3}$ Lynn E. Rose and Raymond C. Vaughan, "Section II: The Artificial Insertion", Kronos, V: 4 (Summer, 1980), pp. 32-47.

${ }^{4}$ William James Douglas has reported on several of these puzzling parallels, but without resolving them. See his "Mayan and Babylonian Observations of Venus", Kronos, VII: 3 (Spring, 1982), pp. 90-91. (In line 5 up on page 90 , for "Section II", read "Section I".) 


\section{Changes in the albedo}

In June of 1993, at the annual meeting of the International Society for the Comparative Study of Civilizations (ISCSC), Dr. Stuart W. Greenwood read a paper on the parallels between the Babylonian and the Maya materials. His starting-point was the empirical fact that there is a near commensurability between the rotation of Venus and the mean synodic period of Venus. He also pointed out that if in the past Venus had a lower albedo than now, then the periods of invisibility would have been longer. Finally, he demonstrated that if the atmosphere of Venus was more transparent than now, if the surface of Venus had varying albedos (perhaps even with oceans as well as land), and if the appropriate parts of the surface were turned toward Earth at the right times during the synodic period, then the periods of visibility as the Evening Star and as the Morning Star would indeed have been different: a darker face would have been turned toward us as Venus entered superior conjunction invisibility, and the invisibility would have begun earlier; likewise, a brighter face would have been turned toward us as Venus emerged from superior conjunction invisibility, and the visibility would have begun earlier. 5

\section{The great dark spot}

I was the commentator on Greenwood's paper at the ISCSC conference. I found no fault at all with his paper, but in the course of my remarks I did mention still another possibility, which had been suggested to me by Raymond C. Vaughan when I told him about Greenwood's idea. Vaughan's suggestion was that Venus need not have had a transparent atmosphere, or a variable surface albedo; it would have been enough if it had had a permanent atmospheric feature, along the lines of whatever Jupiter's Red Spot is. Such a spot could have had a much lower albedo than the rest of the cloud cover. When the spot was turned toward us, Venus would have been harder to see, thus lengthening any period of invisibility that was bounded in that vicinity. But when the spot was turned away from us, Venus would have been easier to see, thus shortening any period of invisibility that was bounded in that vicinity.

Either way, Greenwood's or Vaughan's, various peoples of the past could consistently have seen the Evening Star for a longer period of time than the Morning Star. And either way, Greenwood's or Vaughan's, the atmosphere of Venus would presumably have to have experienced some rather drastic physical changes in the meantime.

\section{The windows theory}

In February of 1995 , I found a radically different way of understanding the Maya Venus numbers.

This new approach involves rejection of the usual interpretation, according to which the 236 days is the period of visibility of the Morning Star, the 90 days is the period of invisibility at superior conjunction, the 250 days is the period of visibility of the Evening Star, and the 8 days is the period of invisibility at inferior conjunction. But the Codex Dresden makes none of these identifications anyway! Such identifications would appear to be entirely in the eye of the beholder.

5 Greenwood's ISCSC paper was entitled "An Interpretation of Mayan Venus Visibility Data"; it remains unpublished but two of his earlier publications that argued along similar lines can be found in the references. 
Suppose instead that the 8 days is a window of opportunity, a span of time somewhere within which the appearance of Venus as the Morning Star might be expected. That was the major event of interest to the Maya, and they needed to know when to look for it. Their 8 days here could even have been intended as an exact figure, neither a mean nor a median.

Suppose also that the 90 days is another window of opportunity, somewhere within which an entire invisibility at superior conjunction would occur. For example, the start of the 90 days might represent the earliest time at which a superior conjunction invisibility could begin, and the end of the 90 days might represent the latest time at which a western visibility could begin. Their 90 days here could likewise have been intended as an exact figure, neither a mean nor a median nor a typical interval nor anything else of the kind. Perhaps they would have used the 90 days to keep track of where they were in the Venus Round. But their principal interest, of course, was the appearance of Venus as the Morning Star.

The 236 days is then to be understood not as the period of visibility of the Morning Star (although there would be a substantial overlap), but merely as the interval between the end of the 8 days and the beginning of the 90 days. Similarly, the 250 days is to be understood not as the period of visibility of the Evening Star (although there would once again be a substantial overlap), but merely as the interval between the end of the 90 days and the beginning of the 8 days.

It has long puzzled people not only that the western interval should be consistently longer than the eastern interval, but also that it should be so much longer. The key to resolving this puzzle of course lies in our recognizing that the 8-day window encompassed only the appearance, and not the disappearance, while the 90 - day window encompassed the superior conjunction invisibility in its entirety - that is, with both ends of the invisibility falling within the window. This inevitably made for a shorter "eastern" period between the 8 days and the 90 days, and for a longer "western" period between the 90 days and the 8 days. If both of the windows had been treated in the same way, and if both of them had been geared to accommodate both ends of their respective invisibilities, then the two windows would have been more symmetrically situated - that is, the "eastern" and "western" intervals separating them would, at least over time, have been approximately equal.

Notice that there is nothing at all in this proposal that would require the visibility of the Morning Star to be substantially shorter than that of the Evening Star! The 236 days and the 250 days are being interpreted quite differently now; no longer are they to be taken as periods of visibility.

Notice also that since the Babylonian figures and the Maya figures are to be explained in two entirely different ways, any resemblance between them must be purely fortuitous. Thus their coincidental similarity should not be taken as evidence of any cultural diffusion - in either direction. (Nonetheless, it must be admitted that if there was contact, then the Maya numbers might have received reinforcement from the knowledge that similar numbers had been used before in Babylonia.)

Notice further that the windows and the Venus numbers take no account at all of the disappearance of Venus near the time of inferior conjunction. Eventually, I found that this disappearance in the west always occurred late in the 250-day period, and never within the 8-day window itself. In any case, disappearance in the 
west seems to have been a matter of relatively little interest to the developers of the windows device and the Venus numbers.

(The Post-Conquest reports about 8 days of invisibility represent a later misunderstanding of the original significance of the " 8 " among the Maya Venus numbers. That misunderstanding seems to have afflicted not only the Spaniards but also the late Maya themselves. It is a misunderstanding that continues to afflict twentiethcentury scholarship as well.)

\section{The time interval}

Over what sort of time interval would this windows arrangement have been intended to operate? At first, and without having done any computations, I thought that windows of 8 days and of 90 days might work fairly well within just a few Venus Rounds. After all, the length of the synodic period of Venus does vary, by a number of days, and the positions of the appearances and disappearances of Venus are also variable. It turned out, however, that both sorts of variations were far too small to fill the windows.

A different approach, and one that I have found to be much more fruitful, takes its start from the Maya correction scheme that is so very sketchily presented on page 24 of the Codex Dresden. (In the numerical illustrations that follow, any quantities that are actually stated on page 24 will be placed in brackets; this is being done merely for the convenience of the reader.)

The Maya realized that 584 days is only the approximate length of the mean synodic period of Venus. In order to anticipate the appearances and disappearances of Venus that interested them, they needed to keep better track of the actual mean synodic period. Hence they developed their correction scheme.

Before we can precisely characterize that correction scheme, however, several of the Maya time units need to be explained.

\section{The time units}

As we have already seen, the period of 584 days is often referred to in the scholarly literature as a Venus Round - or VR for short.

The period of 260 days, which many Mayanists call the $t$ sol k'in (or "day-order"), was actually the basic time-keeping unit throughout much of Mesoamerica. It has also been called the Sacred Round - or SR for short. Another name for it is Sacred Almanac.

The year, or baab, of the Maya was of exactly 365 days, with no intercalation. (The word baab was sometimes applied also to the tun that is, to the Maya period of 360 days. This is potentially confusing, but the overall context usually makes it clear which was meant.)

Another interval that the Maya used was of 364 days. This has been called the Computing Year, the Ritualistic Year, or the Agricultural Count. The plurality of designations may reflect some scholarly uncertainty as to just what the 364-day interval meant for the Maya!

The Calendar Round - or CR for short amounted to 52 of the 365-day years. Each Calendar Round also contained exactly 73 of the Sacred Rounds.

(On the basis of pages 70-74 of the Codex Dresden, Nancy Kelly Owen has recently argued that Maya calendars of $260,360,364$, and 365 days were introduced in succession, and in that very order. ${ }^{6}$ All four of these day-counts then seem to have remained in use, right alongside the others.)

${ }^{6}$ Nancy Kelly Owen, "The Dresden Cosmological Calendars" (forthcoming).
Estudios de Cultura Maya. Vol. XXI, 2000 .

Instituto de Investigaciones Filológicas/

Centro de Estudios Mayas, UNAM 


\section{The correction scheme}

The now-classic explication of the Venus correction scheme was the work of John E. Teeple. ${ }^{7}$ According to Teeple's interpretation, one key feature of the Maya correction scheme was that they deleted 4 days near the end of 61 VR of 584 days each. Since this 4-day correction left them with an integral multiple of 260 , the new VR would begin with 1 'Abaw as its "base" or "day zero", ${ }^{8}$ the same date of the $t$ sol $k^{\prime}$ in upon which the very first of the $61 \mathrm{VR}$ had been based.

The correction had to occur exactly so many days prior to the completion of $2 \mathrm{CR}$. That number of days is $9 \times 26=6.5 \times 360=2340$ :

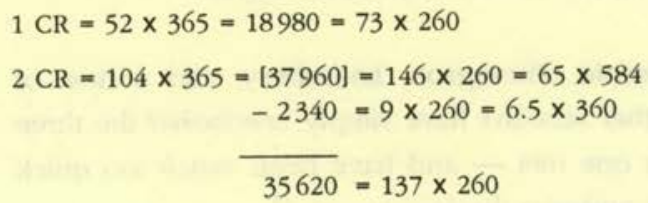

This identifies the new base for the VR, which is the 1 'Ahaw that is just 4 days prior to the end of the $61 \mathrm{VR}$ :

$$
\begin{aligned}
& 35624=61 \times 584 \\
& -4 \\
& 35620=137 \times 260
\end{aligned}
$$

The operation of deleting 4 days near the end of 61 VR was apparently used in conjunction with a quite different operation that involved the deletion of 8 days near the end of 57 VR:

$$
\begin{aligned}
2 \mathrm{CR}=104 \times 365=[37960] & =146 \times 260=65 \times 584 \\
-4680 & =18 \times 260=13 \times 360 \\
\overline{[33280]} & =128 \times 260 \\
33288 & =57 \times 584 \\
-8 & =128 \times 260
\end{aligned}
$$

This second operation would have enabled the Maya to adjust the VR count by 8 days, but again in such a way as to have the new VR based on a 1 'Ahaw.

There is at least some indication that the Maya may have counted a 57 and a 61 together: that is, that they may have proceeded as if the correction cycle included one combined stretch of $57+61=118$ VR. (The 8-day correction for the 57 and the 4-day correction for the 61 would imply a total 12-day correction for the 118.)

118 VR -12 is in fact the same as the "9.11.7.0" that we find on page 24 of the Codex Dresden. This latter expression (which does seem to have some special significance there) is to be interpreted as follows:

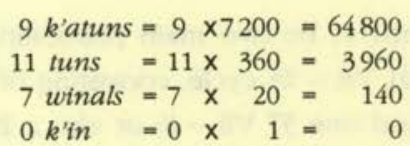

[68900]

The 12-day correction would thus occur near the end of $118 \mathrm{VR}$ :

7 John E. Teeple, "Maya Inscriptions: The Venus Calendar and Another Correlation", American Anthropologist, XXVIII: 2 (April, 1926), pp. 402-408. See also John E. Teeple, Maya Astronomy, pp. 95-96.

${ }^{8}$ See Floyd G. Lounsbury, "The Base of the Venus Table of the Dresden Codex, and its Significance for the Calendar-Correlation Problem", in Aveni and Brotherson, Calendars in Mesoamerica and Peru: Native American Computations of Time, pp. 1-26. 


$\begin{aligned} 4 \mathrm{CR}=208 \times 365=[75920] & =292 \times 260=130 \times 584 \\ \frac{-7020}{[68900]} & =27 \times 260=19.5 \times 360 \\ {[68912} & =118 \times 584 \times 260 \\ -12 & =265 \times 260\end{aligned}$

As before, the commensurability with 260 ensures that the next VR will be based upon a 1 Ahaw.

At this point, it is still not entirely clear whether we are to think of a 57-VR stretch and a $61-\mathrm{VR}$ stretch, each with its own correction, or of a 118VR stretch, with just a single correction near the end. On another occasion, I shall argue that they used a 57-VR stretch and a 61-VR stretch, each with its own correction, and that they only considered using a combined 118-VR stretch with a single correction.

Either way, presumably, the overall length of the correction cycle would have been unaffected. But what was the length of that cycle?

\section{The length of the correction cycle}

There seems to be two main possibilities here: either a 301 VR - 24 cycle, consisting of four 61 VR - 4's and one 57 VR - 8; or else a $240 \mathrm{VR}-$ 20 cycle, consisting of three $61 \mathrm{VR}-4$ 's and one $57 \mathrm{VR}-8$. A $61 \mathrm{VR}-4$ is sometimes referred to as a "single" correction and a $57 \mathrm{VR}-8$ as a "double" correction. Those two main possibilities would then be four singles to one double, or else three singles to one double. (This way of phrasing it of course assumes that the Maya did not use a combined stretch of 118 VR -12 days.)

The 301 VR -24 does work out rather well: $57+61+61+61+61=301$ VR; $584 \times 301=$ 175,784 days; $8+4+4+4+4=24$ days; $175,784-24=175,760$ days; and $175,760-301=$ just under 583.920266 days. But this result may not be significantly different from what we get with 240 VR -20 . After all: $57+61+61+61=$ $240 \mathrm{VR} ; 584$ × $240=140,160$ days; $8+4+4+4$ $=20$ days; $140,160-20=140,140$ days; and $140,140-240=583.916667$ days!

Teeple, Thompson, Lounsbury, and a host of other scholars have simply overlooked the three to one mix - and have been much too quick to embrace the four to one. ${ }^{10}$

I do not yet see any way to determine whether the Maya used three singles or four. But the important point to be noted is that four to one is definitely not an optimum mix.

Perhaps the main reason that Teeple, Thompson, and Lounsbury went astray here is that they were using too rough a value for the mean synodic period of Venus. If the mean synodic period is taken to be 583.92 , then the 301 does work much better than the 240 . But modern figure for the mean synodic period of Venus is

9 Either the 583.920266 or the 583.916667 would compare very favorably with the 583.93405 of Claudius Ptolemy, or even with the 583.9216 of Nicolaus Copernicus. Actually, the Maya seem to have come closer than either of those Old World luminaries! And they accomplished this without the use of fractions! (Quite apart from their non-use of fractions, however, the windows theory itself suggests a certain disdain for means: after all, a window shows us the days on which to look; a mean value does not. Nonetheless, the Maya correction scheme for the Venus Round does seem to imply a mean synodic period of either 583.920266 or of 583.916667 days - depending upon whether they used 301 VR or 240 VR.)

${ }^{10}$ See, for example, Floyd Lounsbury, "A Solution for the Number 1.5.5.0 of the Mayan Venus Table", in The Sky in Mayan Literature, edited by Anthony F. Aveni (New York and Oxford: Oxford University Press, 1992), p. 209: "Thompson noted that the optimum mix of corrections is four singles to one double". 
just under 583.916 days. With a mean synodic period in that vicinity, the 240 , or three to one, is clearly the better of the two mixes.

At this point, I am of course not trying to prove that the Maya did or did not use this or that mix. I am simply stressing that Teeple, Thompson, and Lounsbury have jumped the gun in attributing a unique status to the 301 .

The adherence to 1 'Abaw and to the multiples of 4 were major encumbrances for the Maya. For example, it would have been mucb smoother (and perhaps even more accurate) to jump back 5 days every $61 \mathrm{VR}$, regardless of the dates, and then to jump back 6 days following every eighth stretch of 61 VR. After all, $8 \times 61=488$ VR; 488 X $584=284992$ days; $7 \times 5+6=41$ days; $284992-41=284951$ days; and $284951 \div 488$ $=583.9159836$ days.

Despite such available opportunities, the Maya were quite committed to their 1 'Abaw and to their multiples of 4 , and they were willing to pay a certain price for keeping them. Compared to the scheme in the previous paragraph, their actual practices involved a considerable loss of smoothness. Nonetheless, their combination of 57 VR - 8's and 61 VR - 4's does seem to have worked out very well for them, especially in view of their adherence to 1 'Ahaw and to the multiples of 4 .

The Maya correction scheme for the mean synodic period of Venus would have been operative anywhere on Earth. The point is that almost any Earth-based observer, even one in the polar regions, would find just about the same result for the mean synodic period of Venus; this sort of determination is something that is virtually independent of the observer's terrestrial location, at least over the long run.

The Venus numbers themselves have a quite different status: for they are, at least in part, a reflection of local circumstances, not only in that they depend upon the latitude of the observation-post, but also in that they depend upon the arcus visionis. (The arcus visionis of a given body is traditionally defined as the minimum angle below the horizon that the Sun must be in order for the given body, in this case Venus, to be visible on the horizon.)

The remarkable accuracy of the Maya concerning the mean synodic period of Venus only deepens our puzzlement over their Venus numbers. After all, these are the very same people who had been using negative numbers, place-values, and zero - arguably even before there were any such developments in the Old World. If the Maya could lead all other peoples both in astronomical accuracy and in the science of arithmetic, how could they make such a mess of their own Venus numbers?

Perhaps (to paraphrase Shakespeare), the fault is not in them - or in their stars - but in ourselves.

\section{Using the computer}

In order to determine just how well the windows theory worked - and, more specifically, in order to see whether the Maya Venus numbers might be the natural outcome of a stretch of $61 \mathrm{VR}-$ I needed a long sequence of disappearances and appearances of Venus. Fortunately, Raymond C. Vaughan's program V87ARC could be adapted

Lounsbury cites Thompson's Maya Hieroglyphic Writing (1950), p. 227a, and his own "Maya Numeration, Computation, and Calendrical Astronomy", pp. 788-789. Lounsbury might also have cited p. 783 of his own paper, as well as Teeple, "Maya Inscriptions: The Venus Calendar and Another Correlation", p. 404, who had said the same thing back in 1926. (See also Teeple's Maya Astronomy, 1931, p. 96.) 
for this particular application, and could be set for whatever latitudes and epochs might seem appropriate for what the Maya saw.

Using a modification of Vaughan's program, I checked the arcus visionis values from $5.0^{\circ}$ to $10.0^{\circ}$, in increments of $0.5^{\circ}$, and I checked the latitudes from $13.0^{\circ}$ to $21.5^{\circ}$, again in increments of $0.5^{\circ}$. This was over a stretch of $61 \mathrm{VR}$, and with an approximate starting epoch of +1100 .

These initial runs were very encouraging: they often came quite close to the actual Venus numbers, sometimes even bitting one or two of them. But the initial runs failed to turn up any eastern appearance window of fewer than nine days. (Only much later did I find just a few eastern appearance windows that were one day shorter than this.)

Almost at once, I began to suspect that I had been using some interval other than what the originators of the Venus numbers had had in mind. I needed to retbink the nature of the intervals that I had been looking for. This problem is of course complicated by the fact that the Maya did not use fractions.

I had been counting the number of whole days on which it would have been appropriate to look for the appearance of the Morning Star. But the looking itself was always at dawn. Thus the rising of the Morning Star could have been handled in a quite straightforward manner simply by counting from one dawn to a later dawn: after all, the actual interval from, say, dawn on day 1 to dawn on day 9 is only eight days. Viewed in this way, the window is only eight days long.

In a similar manner, the "eastern" period could then be measured from the dawn that marks the end of the inferior rising window to the dawn that marks, say, the earliest first invisibility. (There are no whole day problems here, but it remains an open question whether we Estudios de Cultura Maya. Vol. XXI, 2000

Instituto de Investigaciones Filológicas/

Centro de Estudios Mayas, UNAM

ISSN: 0185-2574 should be using the earliest last visibility or the earliest first invisibility. Any eventual decision about this issue will in turn affect the length of the superior window.)

At least as a provisional conjecture, let us assume that they took the 236 to have ended with the earliest first invisibility, that is, on the day after the earliest last visibility. This can always be reconsidered if the computations suggest otherwise. In the meantime, the program will use earliest first invisibility as the dividing line between the 236 and the 90 , and we need to be looking for 236 and 90 as the first two Venus numbers. But 237 and 89 would also be acceptable. For if the Maya had used earliest last visibility as the dividing-line, then what the computer counts as $237-89-250-8$ would have been seen by the Maya as $236-90-250-8$ ! It is by no means obvious whether they would have used earliest last visibility or earliest first invisibility as their criterion here.

The termini for the heliacal rising window after inferior conjunction are relatively straightforward now: the window ran from earliest first visibility to latest first visibility. It is the superior window whose termini remain problematic. Presumably the superior window could have run either from the dawn of the earliest last visibility in the east or from the dawn of the earliest first invisibility in the east, and could have ended either with the dusk that marked the latest last invisibility in the west or with the dusk that marked the latest first visibility in the west.

If they used earliest first invisibility as the start of the 90, and if they used latest first invisibility as the end of the 90 , then what the computer counts as 236-91-249-8 would also have to be considered acceptable, since this too would have been seen by the Maya as 236-90-250-8.

But if they used earliest last visibility as the start of the 90 , and if they used latest last invis- 
ibility as the end of the 90 , then what the computer counts 237-90-249-8 would likewise have to be considered acceptable, since this as well would have been seen by the Maya as $236-90$ 250-8.

What about the problem of whole days here?

The 90-day window seems to have extended from a dawn to a dusk. Strictly speaking, then, must not the "90 days" have been $89 \frac{1}{2} /$, or perhaps $90 \%$ ? And since we presumably do need to avoid the half-days in such situations, ought we to round up or round down?

The "western" period begins either with the dusk that marks the latest last invisibility or with the dusk that marks the latest first visibility, and runs to the dawn that marks the start of the heliacal-rising-of-Venus window. Notice that we are now moving from a dusk to a dawn. Once again, we need to avoid the half-days, but are we to do this by rounding up or by rounding down?

Since the 90 and the 250 are reported in whole days, and since the sum of the four Venus numbers is 584 , it is clear that one of those two intervals must have been rounded $u p$ and the other down. But which is which? I suggest that they rounded $u p$ to the 90 and down to the 250. After all, rounding a window down would run the risk of making it too small to serve as the intended sort of window. I set V87ARC accordingly.

Let us review what we are looking for. There are four acceptable results:
(A) $236-90-250-8$
(B) $237-89-250-8$
(C) $236-91-249-8$
(D) $237-90-249-8$

These four cases differ according to the demarcation of the 90 , which could have been counted in any of these four ways:
(A) from earliest first invisibility to latest first visibility

(B) from earliest last visibility to latest first visibility

(C) from earliest first invisibility to latest last invisibility

(D) from earliest last visibility to latest last invisibility

These four cases may not be equally plausible, but at this point there is no way to eliminate any of the four.

It had of course been clear from the very first runs that I was indeed looking in the right place now - that is, over a stretch of 61 VR -4 days. Even though some of the numbers were off especially the 9 instead of 8 , which was a serious problem until I corrected my way of thinking about the heliacal rising window - I had usually been able to locate one or two of the four Venus numbers rather easily. (It is possible to hit all four, or none, or one or two, but never exactly three: for if one of the numbers is off, then at least one of the otber numbers will also be off simply because the total of the four numbers has to be 584.)

After making the various alterations and corrections that have just been discussed, I checked the arcus visionis values from $5.0^{\circ}$ to $10.0^{\circ}$, with increments of $0.5^{\circ}$, and the latitudes from $9.0^{\circ}$ to $22.0^{\circ}$, but with increments of $1.0^{\circ}$ now (I had recognized after just a few runs that the outcomes are considerably less affected by the latitude than by the arcus visionis; I had also recognized that $13^{\circ}$ was too high a startingpoint, and that I would have to sample some still lower latitudes as well.)

The best rough values seemed to be with an arcus visionis of something above 9.0 and a lati- 
tude of around $9.0^{\circ}$ to $14.0^{\circ}$ (or perhaps even $\left.15.0^{\circ}\right)$. I then adjusted the program to test the arcus visionis values from $9.0^{\circ}$ to $9.7^{\circ}$, with increments of $0.05^{\circ}$, and to test the latitude values from $8.0^{\circ}$ to $16.0^{\circ}$, with increments of $0.1^{\circ}$.

Now, with an arcus visionis of $9.15^{\circ}$ to $9.6^{\circ}$ and a latitude of from $8.8^{\circ}$ to $13.9^{\circ}$, and with a stretch of 61 Venus Rounds, V87ARC yielded 146 acceptable results. (I shall sometimes refer to these as "pairs", since each acceptable result does consist of a pair of numbers, one for the arcus visionis and one for the latitude.) Of the 146 acceptable pairs, 57 were 237-90-249-8, or (D), extending from $8.8^{\circ}$ to $11.6^{\circ}$. No $236-91-249-8$ 's, or (C)'s, were found for +1100 . 75 were $237-89-250$ 8 , or (B), extending from $10.6^{\circ}$ to $13.4^{\circ} .14$ were 236-90-250-8, or (A), which were scattered rather thinly from $12.1^{\circ}$ to $13.9^{\circ}$. As was explained earlier, any of these four would be acceptable.

In this large group of acceptable combinations, there was a pronounced tendency for the $237-90$ 's to occur to the south and for the 236 90 's to occur to the north, with the 237-89's lying in the middle and overlapping the latitudes of the other two. The 237-90's and the 237-89's were much more numerous than the 236-90's $(57$ and 75 , versus only 14 ), even though the 23690's were a little closer, at least, to the traditionally-recognized locale of the Maya.

So far, the evidence might seem to favor one of the 237's, especially if lower latitudes were involved. But we still have no proof that earliest last visibility was the line of demarcation between the 236 and the 90 . Besides, the Maya Venus numbers may well have been derived many centuries earlier, when the astronomical circumstances were at least slightly different.

In two respects, our results up to this point may be a little surprising. After all, $8.8^{\circ}$ to $13.9^{\circ}$ is perhaps a rather low range for the latitude, and $9.15^{\circ}$ to $9.6^{\circ}$ is perhaps a rather bigh range for Estudios de Cultura Maya. Vol. XXI, 2000 the arcus visionis. Let us discuss each of these questions in turn.

\section{The latitude}

It is easy to see why latitude is so important to us: once we find the latitudes that make for the best fit, that is, the latitudes that best explain or best duplicate the Venus numbers, we might then be able to make a plausible identification of the site to which those Venus numbers originally pertained. This would not prove that the Codex Dresden and/or the Codex Grolier originated at that site, but it would suggest that the observations themselves might have been made at that particular site.

That is why our low latitudes are rather surprising. We might rather have expected the provenance of the Venus numbers to be much more to the north, perhaps even in the Yucatan. Instead, we are finding latitudes that pass through Guatemala, El Salvador, and Honduras, as well as other latitudes that pass through Nicaragua and even Costa Rica. ( $8.8^{\circ}$ would put us in southern Costa Rica, or even in northern Panama!)

We must, of course, be prepared to distinguish such things as the site(s) of the original $o b$ servations, the site(s) where those observations were studied, the site(s) where a codex was written, and the site(s) where a codex was used. In any case, we cannot exclude an original observation-post at one of those lower latitudes, especially in earlier times. But it remains to the seen whether those earlier times are equally suggestive of lower latitudes.

\section{The arcus visionis}

The values for the arcus visionis are a bit bigh$e r$ than we might have expected. 
A general elevation of arcus visionis values for Venus could of course be attributed to conditions on Venus. Both the surface and the atmosphere of Venus may have been rather turbulent over, say, the last few millennia. That is why some might be tempted to infer that Venus did once have a significantly lower albedo back whenever it was that the Maya were making the observations that led to their Venus numbers.

But the actual causes of those higher arcus visionis values are most likely to have been terrestrial circumstances, namely, obstructed views and misty horizons - the very sorts of things that might be expected in Mesoamerica. ${ }^{11}$

\section{The Early Classic Period}

Could any better results have been obtained, if an epoch in the Early Classic Period had been chosen instead? So far, I had been using +1100 as the approximate epoch. Now I decided to try +400 . (This 700-year spacing between trials is entirely arbitrary.)

With +400 , the best rough values seemed to be with an arcus visionis of between $9.2^{\circ}$ and $9.7^{\circ}$ and a latitude of around $11.0^{\circ}$ to $15.0^{\circ}$. I then adjusted the program to test the arcus visionis values from $9.0^{\circ}$ to $9.8^{\circ}$, with increments of $0.05^{\circ}$, and to test the latitude values from $10.0^{\circ}$ to $16.0^{\circ}$, with increments of 0.1 .

Now, with an arcus visionis of $9.2^{\circ}$ to $9.65^{\circ}$ and a latitude of from $10.1^{\circ}$ to $15.5^{\circ}$, and with a stretch of 61 Venus Rounds, V87ARC yielded 229 acceptable results. Of these, 48 were 237 90-249-8, or (D), extending from $10.1^{\circ}$ to $12.5^{\circ}$. 78 were 236-91-249-8, or (C), extending from $11.1^{\circ}$ to $13.8^{\circ}$. 26 were $237-89-250-8$, or (B), extending from $12.3^{\circ}$ to $14.2^{\circ}$. And 77 were 236 $90-250-8$, or (A), extending from $13.0^{\circ}$ to $15.5^{\circ}$. As was explained earlier, all four of these are acceptable.

In this large group of acceptable combinations, there was a pronounced tendency for the $237-90$ 's to occur to the south and for the 23690 's to occur to the north, with the 236-91's and the 237-89's lying in the middle and overlapping the latitudes of the other two. From south to north, the order was $237-90,236-91,237-89$, and 236-90, that is, (D), (C), (B), and (A). The 23690 's and the 236-91's were more numerous than the $237-89$ 's and the $237-90$ 's ( 77 and 78 versus 26 and 48), with the 236-90's lying a little closer, at least, to the traditionally-recognized locale of the Maya.

On this basis, we might be tempted to infer that the dividing-line between the 236 and the 90 was the earliest first invisibility (as the program assumes anyway), rather than the earliest last visibility. The observations upon which the Maya Venus numbers were based might then have been made in the Early Classic Period, perhaps at a latitude in the vicinity of, say, $12.0^{\circ}$ to $15.0^{\circ}$. The arcus visionis in excess of $9.0^{\circ}$ suggests a jungle or mountain setting, perhaps in Guatemala or Honduras, or even in El Salvador or Nicaragua.

${ }^{11}$ On page 87 of "Maya Astronomy", Thompson mentions "the hindrances to accurate observation caused by cloudy weather in the rainy season and morning mist in the dry season". He does not elaborate, but he may be hinting at factors that would impede morning - that is, eastern - seeing. These considerations are not nearly enough, however, to explain so substantial a difference as $250-236=14$. (V87ARC reveals that the western and eastern values of the arcus visionis would have to differ overall by something like three degrees in order to have this effect. Besides, Thompson's "hindrances" would be essentially seasonal. Thus the actual over-all difference between western and eastern visibilities would have to be averaged out over all of the other times of the year, and would be somewhat attenuated.) 
But we should not be so quick to jump to any such conclusions. There are still other possibilities that must be taken into account.

\section{The Pre-Classic period}

I next checked -300 , which would have been in the so-called "Pre-Classic" Period.

With -300 , the best rough values seemed to be with an arcus visionis of somewhere around $9.0^{\circ}$ or $9.5^{\circ}$ and a latitude of from about $11.0^{\circ}$ to about $16.0^{\circ}$. I then adjusted the program to test the arcus visionis values from $9.0^{\circ}$ to $9.6^{\circ}$, with increments of $0.05^{\circ}$, and to test the latitude values from $10.0^{\circ}$ to $17.0^{\circ}$, with increments of $0.1^{\circ}$. (The $9.0^{\circ}$ later had to be lowered to $8.7^{\circ}$, and the $17.0^{\circ}$ had to be raised to $18.5^{\circ}$.)

Now, with an arcus visionis of from $8.75^{\circ}$ to $9.5^{\circ}$ and a latitude of from $11.5^{\circ}$ to $18.4^{\circ}$, and with a stretch of 61 Venus Rounds, V87ARC yielded 263 acceptable combinations.

This time, 54 were $237-90-249-8$, or (D), extending from $11.5^{\circ}$ to $13.5^{\circ}$. 58 were $236-91-249$ 8 , or (C), extending from $12.5^{\circ}$ to $14.8^{\circ} .57$ were 237-89-250-8, or (B), extending from $13.1^{\circ}$ to $15.3^{\circ}$. And 94 were $236-90-250-8$, or (A), extending from $14.0^{\circ}$ to $18.4^{\circ}$.

$18.4^{\circ}$ is the last fit in a long, tapering trail-off; the thick part of $236-90$ is from $14.7^{\circ}$ to $16.1^{\circ}$ much more to the south. Even though there is nothing decisive in this regard, the overall thrust of our findings is still very much toward the south: the median latitude for all 263 cases is only $14.1^{\circ}$.

In this large group of acceptable combinations, there was again a pronounced tendency for the 237-90's to occur to the south and for the 236-90's to occur to the north, with the 236-91's and the 237-89's lying in the middle and overlapping the other two. From south to north, the order was once again $237-90,236-91,237-89$, and 236-90, that is, (D), (C), (B), and (A). The 236-
Estudios de Cultura Maya. Vol. XXI, 2000
90 's, or (A)'s, were not only much more numerous than the others ( 94 for $(A)$ versus 57,58 , and 54 for (B), (C), and (D), respectively), but were again somewhat closer, at least, to the traditionally-recognized locale of the Maya.

The overall picture is like this:

Post-Classic: 146 hits, $9.15^{\circ}-9.6^{\circ}$ and $8.8^{\circ}-13.9^{\circ}$ Early Classic: 229 hits, $9.2^{\circ}-9.65^{\circ}$ and $10.1^{\circ}-15.5^{\circ}$ Pre-Classic: 263 hits, $8.75^{\circ}-9.5^{\circ}$ and $11.5^{\circ}-18.4^{\circ}$

This abundance of riches is reassuring, but it leaves us with very little basis for picking out the approximate epoch at which the observations might have occurred. It also leaves us with very little basis for resolving our uncertainties about the lines of demarcation.

The following is a convenient simplification of the overall pattern of Pre-Classic hits. The arcus visionis values (across the top) are given in increments of $0.05^{\circ}$, just as in the program, but the latitudes (down the side) are given in increments of $0.5^{\circ}$, instead of the $0.1^{\circ}$ of the program. (A), (B), (C), and (D) of course represent the four possible sets of boundaries for the 90-day interval. (That some of these letters are in lower case is unimportant for now; this is something that will be explained in the Appendix.)

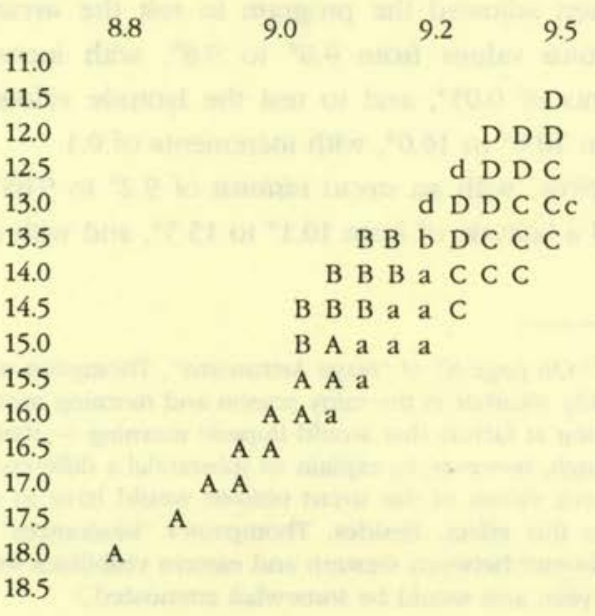


One of the more significant results here is that the hits are concentrated between latitude $13.0^{\circ}$ and latitude $15.0^{\circ}$. Once again, the implication is that the Maya sphere of activity may have extended farther south than most would have expected. This is something that Nancy Kelly Owen has been saying for years! She and I have arrived at that perspective from entirely different directions, and for entirely different reasons. That in itself suggests that we may both be correct! ${ }^{12}$

\section{The site?}

Before closing, I wish to emphasize that the many pairs of arcus visionis values and latitudes, as well as epochs, are but possibilities that meet the requirements that we have set. The Maya's own decisions about the boundaries of the 90-day window would have determined whether they were in the (A), (B), (C), or (D) mode. But they did not actually choose their epoch; neither did they actually choose a latitude or an arcus visionis, except in the sense that when they chose their observation-post, they were also getting the latitude and the arcus visionis that pertained to that site. In any event, presumably no more than one of the many possibilities that we are considering would actually have corresponded to historical reality here!

\section{Conclusion}

The Maya Venus numbers that have always been dismissed as non-empirical and even weird now turn out to make quite good sense!
They seem to have encapsulated a vast amount of observational and empirical material. It is surely no accident that we are now finding that there is every likelihood that, over the course of $61 \mathrm{VR}$, the Maya Venus numbers were accurate to the very day!

Acknowledgements. I am grateful both to Nancy Kelly Owen, for her generous and helpful advice regarding Maya studies generally and Maya astronomy in particular, and to Raymond C. Vaughan, for the use of his Fortran program V87ARC, which has enabled me to make such a very strong case for the exactitude of the Maya Venus numbers. Computer work was done on the IBM mainframe at the State University of New York at Buffalo.

\section{APPENDIX}

\section{Some suggestions about the tsolk'in}

The 260-day period called the $t$ sol k'in has long been a popular subject for creative exegesis; it is fair to say, however, that most of the resulting interpretations have been considered both speculative and strained, even by their authors! ${ }^{13}$

I now find that I have some suggestions of my own to add to the long list. I offer them only tentatively, and with a certain trepidation.

What might we ask of a plausible interpretation of the tsol k'in? Well, it should reflect some natural period, preferably astronomical; it should at least come close to 260 days; it should be fairly simple; and it should involve events that

${ }^{12}$ Nancy Kelly Owen, "The Dresden Cosmological Calendars" (forthcoming).

${ }^{13}$ One recent such attempt is by John W. Burgess, "A Possible Astronomical Genesis of the Tzolkin", in Iwaniszewski et al., Time and Astronomy at the Meeting of Two Worlds, pp. 60-71. 
would have been awesome enough to have commanded the attention of the Maya in the first place. (Some might also want Venus involved.)

There are three sorts of 260-day intervals to which I wish to call attention. For purposes of illustrating these three sorts of intervals, we need only refer to the detailed samplings that I have already made, where $+1100,+400$, and -300 were the approximate epochs.

Just one of the three sorts of intervals occurs in the Post-Classic Period (as represented by +1100 ). All three do occur in the Early Classic Period (as represented by +400 ), but only rather infrequently. I could use the Early Classic Period here, but I prefer to concentrate instead on the Pre-Classic Period (as represented by -300), where the pickings are much richer. Not only do all three sorts of 260-day intervals occur in the Pre-Classic Period, but at least two of them tend to occur much more frequently there than in the Early Classic Period. (Also, it seems to be only in the Pre-Classic Period that all three of them can occur together.)

We have already seen that the Maya had an 8day window for the first visibility of the Morning Star. This window extended from the earliest first visibility of the Morning Star to the latest first visibility of the Morning Star. Let us now imagine that the Maya also had a window for the first visibility of the Evening Star. (There is no direct evidence of this, but let us just suppose.) With the Morning Star window as a model, it seems very likely that an Evening Star window would have extended from the earliest first visibility of the Evening Star to the latest first visibility of the Evening Star.

We have still not been able to establish whether the 90-day window for the superior conjunction invisibility began with the earliest last visibility or

Estudios de Cultura Maya. Vol. XXI, 2000

Instituto de Investigaciones Filológicas/

Centro de Estudios Mayas, UNAM

ISSN: 0185-2574 with the earliest first invisibility, or whether it ended with the latest last invisibility or with the latest first visibility. If we do have an Evening Star window, then latest first visibility might be more attractive for the ending of the 90-day window, in that the 90-day window and the Evening Star window would then end at precisely the same point. But latest last invisibility is also feasible: the Evening Star window would in that case simply have to extend one day later than the 90-day window.

Still another possibility is that the Maya had a window for the last invisibility. (A last invisibility window would of course not only end but also begin one day earlier than an Evening Star window.) If the 90-day window ended with the latest last invisibility, then both the last invisibility window and the 90-day window would have ended at precisely that same point. (If the 90-day window ended with the latest first visibility, however, then a last invisibility window would have to have ended one day before the 90-day window ended.)

Juggling these various new possibilities while trying to discuss the tsol $\mathrm{k}^{\prime}$ in will prove somewhat awkward; after all, we are already juggling (A), (B), (C), and (D) - our four possible readings of the Maya Venus numbers. In order to simplify the ensuing remarks, I need to be able to lump some of those newer possibilities together, especially the last invisibility window and the Evening Star window.

With deliberate imprecision, then, I shall be speaking of a "western window". This could be either a last invisibility window or an Evening Star window, indifferently. I shall also be speaking of a "western event", which could be either a last invisibility or a first visibility, indifferently.

V87ARC discloses that, among our 263 hits from the Pre-Classic epoch, there are eight western 
windows of 11 days and 51 western windows of 9 days; the remaining 204 western windows are of 10 days. These 204 instances of 10-day windows are divided about equally among our four possibilities: (A) has 54, (B) has 51 , (C) has 52 , and (D) has 46. (The solutions with western windows of either 9 days or 11 days were the ones that were put in lower case in our earlier diagram.)

The "western" period, the period between the 90-day window and the 8-day window, is of course 250 days long. And $10+250=260$. Provided that we are considering a first visibility window together with either (A) or (B), or else a last invisibility window together with either (C) or (D) - in other words, provided that the 90-day window and the 10-day western window end together - the interval between the start of the western window and the start of the Morning Star window is 260 days, exactly. If the western window begins on, say, $10 \mathrm{Kib}$, so also does the Morning Star window begin on $10 \mathrm{Kib}$ !

Taken in the abstract, a last invisibility window may seem somewhat less plausible than a first visibility window. (After all, the latter involves much more of a spectacle than the former.) But if we do once recognize that a last invisibility window could have allowed the Maya to accommodate the 260 in the manner just indicated, then the idea that they might have used a last invisibility window suddenly seems very plausible indeed!

Even if the Evening Star window does $=9$, as in 51 of the (A), (B), and (C) pairs, it is noteworthy that $9+(250+1 \Rightarrow 251=260$. The point is that if the 90-day window ends with the latest first visibility, as in all of the (A) and (B) pairs, then even if the Evening Star window does slip to 9, the interval between the start of the last invisibility window and the start of the
Morning Star window would still be 260 . (This does not apply to (C), where the 90-day window does not end with the latest first visibility.)

And even if the Evening Star window does = 11 , as in eight of the (D) pairs, it is noteworthy that $11+(250-1 \Rightarrow 249=260$. The point is that if the 90-day window ends with the latest last invisibility, as in all of the (C) and (D) pairs, then even if the Evening Star window does rise to 11 , the interval between the start of the Evening Star window and the start of the Morning Star window would still be 260 days. (This does not apply to (C), which has no 11's.)

Thus if we do want to get to the 260 here, we cannot rule out either the 9's or the 11's except where (C) is involved. Even if the original observations had been made at one of those 59 locations where the western window was 9 days or 11 days, the right sort of boundary at the end of the 90-day window could still have saved the 260 - except where (C) was involved.

In all of these favorable cases, we have an astronomical interval of exactly 260 days. Furthermore, our account of the circumstances is both simple and natural. It even involves Venus - whose behavior was of such profound importance to the Maya! What remains problematic, however, is whether this occurrence of a 260-day interval would be enough to explain the origins of the tsol k'in. (Later, I shall argue that it would not be enough.)

If it is this easy to come up with 260 here, why haven't others already done it? Because they were not working with the windows interpretation of the Maya Venus numbers. The idea of counting from the starting-date of one window to the starting-date of another window simply never crossed their minds. 
As we shall see, the other two intervals of 260 days that I wish to discuss have also been overlooked. Here, too, the reason that no one else found them is that no one else was working with the windows interpretation of the Maya Venus numbers. As a consequence, no one ever checked the right arcus visionis values or the right latitudes. (The right ones, of course, are the ones that generated the Maya Venus numbers for us in the first place!)

Astronomical analyses and astronomical writings tend to be from the perspective of the north temperate zone. It is all too easy for people to suppose that what they see now from the north temperate zone was what the Maya saw then from the tropics. In any case, even if someone had checked, using latitudes that were too high and arcus visionis values that were too low, the interval between the Evening Star and the Morning Star would still have to have been whatever the visibility of the Evening Star was taken to be, plus whatever the inferior invisibility amounted to. Thus the Evening Star to Morning Star interval would have climbed at least into the middle or high 260 's, and more likely into the 270 's or even the lower 280's. That is why the Evening Star to Morning Star interval has routinely been overlooked, as being far too large to be an instance of the 260 days.

But the Evening Star to Morning Star interval was actually much shorter for the Maya than many would nowadays suspect. Taking all of our 263 hits for the Pre-Classic epoch, we find that, over the course of 61 Venus Rounds, the average interval between Evening Star and Morning Star ranges from 258.25 to 259.87 . These numbers are a far cry from, say, the 270 's.

If the Maya counted from last invisibility in the west to first visibility in the east, those numEstudios de Cultura Maya. Vol. XXI, 2000

Instituto de Investigaciones Filológicas/

Centro de Estudios Mayas, UNAM

ISSN: 0185-2574 bers would have to be increased by one day, and the average interval between the western event and the Morning Star would then range from 259.25 to 260.87 . This is a second sort of 260-day interval. We can easily pick out those pairs that end up fairly close to 260 . If the actual observation-post did have the latitude and the arcus visionis of one of those pairs, the Maya might very well have noticed that the average interval between the last invisibility in the west and the first visibility in the east was approximately 260 days.

If the Maya counted from the day on which the last invisibility in the west occurred, and if they counted to the day on which the first visibility in the east occurred, then those numbers would have to be lowered by 0.5 days. But if such a reduction by 0.5 days was involved, then our increase by 1.0 days is needed all the more. We would then be looking for an average interval of about 259.5 days between the Evening Star and the Morning Star, that is, between the first visibility in the west and the first visibility in the east: $259.5-0.5+1.0=260.0$.

In order to be able to increase the numbers by 1.0 , we would have to count from the last invisibility rather than from the first visibility. This would presumably put us in (C) or (D), where the 90-day window does end with the latest last invisibility. Unfortunately, V87ARC discloses no averages either in (C) or in (D) that are as high as 259.5. Averages of about 259.5 do occur in (A) and in (B), but they are to no avail, since the 90-day window ends with the latest first visibility in (A) and (B).

Another possibility would be to try to avoid the reduction by 0.5 days. (This could be done by continuing to count from one event to another event, rather than from one day-on-which to another day-on-which.) We would then be looking for an average interval of about 259.0 
days between the Evening Star and the Morning Star, that is, between the first visibility in the west and the first visibility in the east: $259.0+1.0=$ 260.0.

Are there any 259.0 's in (C) or (D)? Yes; there are several of these, but only in (D). One example is with arcus visionis $=9.3^{\circ}$ and latitude $=12.9^{\circ}$. The mean interval from Evening Star to Morning Star is 258.95 days, within a range of 254.5 to 263.5 . If we count from last invisibility rather than from first visibility, the mean interval becomes 259.95 days, within a range of 255.5 to 264.5 . This mean interval of 259.95 days would presumably have been quite close enough for their purposes.

One word of caution here: we should remember that the windows approach, in itself, involves no averages whatsoever, and may actually reflect a certain disdain for averages! Even the Maya correction scheme for the synodic period of Venus only implies that mean value of either 583.920266 or 583.916667 ; the important thing to them was probably not the mean value in itself, but simply that their correction scheme would enable them to keep better track of Venus over the course of time! (Their eclipse tables, likewise, feature opportunities or windows, rather than averages or means.)

Notice that the mean of those two extreme values (255.5 and 264.5) is exactly 260.0 days. While still avoiding the half-day reduction, these circumstances offer the opportunity to recognize a third interval of 260 days. For this purpose, one could again count, not from date to date, or from day-on-which to day-on-which, but rather from one point in time to another point in time - in this instance, from a dusk to a dawn and thus one could land on the mid-point (the mean of the extremes) of the range of intervals between the western event and the first visibil- ity in the east. This mean-of-extremes situation occurs in well over half of our 263 pairs. (The count is sometimes from last invisibility, and sometimes from first visibility.)

The mean of extremes here is undeniably a mean, of course, but it is not a mean of the entire set of values falling within that range. Thus any disdain that the Maya may have felt for means or averages need not have affected this mean of extremes situation, which is more a matter of identifying the middle or mid-point of a window.

After all, a range of, say, 255.5 to 264.5 is, in effect, another window. This time, it is a 9-day window of opportunity whose position is determined by the previous western event; thus its location in the Venus Round could not have been ascertained until the actual position of the western event had been ascertained. Furthermore, its location would shift within the Venus Round.

This shifting window overlaps the customary 8-day window. The latter, of course, could be considered fixed, at least insofar as its intended position was at the very end of the full Venus Round, between day 576 and day 584 . In practice, however, the slippage caused by the fact that the mean synodic period of Venus is slightly less than 584 days would mean that the actual 8day interval within which the heliacal risings took place would occur earlier and earlier within the VR.

When taken together, this de facto heliacal rising window and our mean-of-the-extremes window might permit a further narrowing of the dates on which the heliacal rising of the Morning Star could occur. Notice that for this purpose an earlier western event would be much more enlightening than a later western event. The smaller the overlap of the two windows, the more precise the prediction. Under ideal conditions, and with the range at 256.5 to 263.5 , there 
could then be as few as five dawns among which to choose.

To summarize, we have found three different intervals of 260 days:

(1) Exactly 260 days from the starting-date of the appropriate western window to the startingdate of the Morning Star window.

(2) An average of about 260 days from the last invisibility in the west to the first visibility in the east.

(3) Exactly 260 days from the appropriate western event to the mean of the extremes of the first visibility in the east.

Here and there among our 263 pairs, we do find all three of these intervals at once. Thus our earlier example (which was a case of (D), with arcus visionis $=9.3^{\circ}$ and with latitude $=$ $12.9^{\circ}$ would feature (1), (2), and (3); furthermore, both its 90-day window and its western window would end with the same event, namely, latest last invisibility.

As we have seen, the Maya's own delineation of the boundaries of the 90-day window would determine whether they were in the (A), (B), (C), or (D) mode. Other than that, however, the Maya did not actually choose among our pairs. Presumably they simply chose a site, which of course had a determinate latitude and arcus visionis. Also, that site either did or did not involve a 9-day, 10-day, or 11-day western window, and either did or did not involve some combination of the three 260-day intervals that have just been discussed.

We are simply trying to identify possible pairs. At present, however, there are still far too many of them for us to be able to pick out the pair that describes the actual site from which the original observations were made.

Estudios de Cultura Maya. Vol. XXI, 2000

Instituto de Investigaciones Filológicas/

Centro de Estudios Mayas, UNAM

ISSN: 0185-2574
I do not see any or all of our three 260 's as the likely origin of the tsol k'in. They are simply not weighty enough to account for the profound impact that that number had on the Maya and others. At the very most, they might help explain the continuing Maya preoccupation with the tsol k'in. In other words, these 260 's might well have reinforced a much earlier tradition, but a tradition based on considerations of an entirely different sort.

The tsol k'in was important enough to the Maya that we should not be surprised if the number 260 turns up in more than one place. If the tsol k'in was already hoary and venerated, it is unlikely that the Maya developers of the windows device would have overlooked any obvious opportunities to incorporate 260 's in their model.

Whatever it was that started the tsol k'in must have been very significant and very impressive, in order to be able provide the calendrical linchpin for the whole of Maya civilization - as well as for many of their neighbors, who had also adopted 260 days as a basic time-keeping interval.

The three 260 's that we have found within the context of the windows and the Venus numbers are intriguing, but they are hardly very awesome or profound. There is simply nothing here that has the makings of enduring tradition. The principal appeal that our three 260 's would have had for the Maya is much the same sort of appeal that they have for the reader and that they have for me: namely, the appeal that they would have for anyone who is already preoccupied with the number 260 . It is clear enough how the reader and I acquired that preoccupation: we have an interest in the Maya. How the Maya themselves originally acquired that preoccupation is a question that is simply not to be resolved by way of our investigations here. 


\section{References}

Avenl, Anthony F.

1992 Conversing with the Planets. New York: Times Books.

$\longrightarrow$, editor

1992 The Sky in Mayan Literature. New York: Oxford University Books.

Aveni, Anthony F., and Gordon Brotherson, editors

1983 Calendars in Mesoamerica and Peru: Native American Computations of Time. Oxford: BAR.

BURGESS, JOHN W.

1983 "A Possible Astronomical Genesis of the Tzolkin", in Iwaniszewski et al., editors, Time and Astronomy at the Meeting of Two Worlds, pp. 60-71.

DOUglas, WilLiam James

1982 "Mayan and Babylonian Observations of Venus", Kronos, VII: 3 (Spring), pp. 90-91.

GreenwoOd, StuART W.

1985 "Atmospheric Changes on Venus?", Ancient Skies XII:4 (September-October), pp. 1-2.

1990 "Mayan Pointers to Surface Variations on Venus", Ancient Skies, XVII: 1 (MarchApril), p. 1.

1993 "An Interpretation of Mayan Venus Visibility Data", presented at the International Society for the Comparative Study of Civilizations, Scranton, Pennsylvania, June 4. Unpublished.

Iwaniszewski, Stanislaw, Arnold Lebeuf, Andrzej WierCINSKI, AND MArIUSz S. ZıOL-Kowski, editors

1994 Time and Astronomy at the Meeting of Two Worlds. Warsaw: Center for Latin American Studies (CESLA), Warsaw University.

LOUNSBURY, FLoYd G.

1978 "Maya Numeration, Computation, and Ca- lendarical Astronomy", in Dictionary of Scientific Biography, edited by Charles C. Gillispie. Volume XV (Supplement), pp. 759-818. New York: Charles Scribner's Sons.

1983 "The Base of the Venus Table of the Dresden Codex, and its Significance for the Calendar-Correlation Problem", in Aveni and Brotherson, Calendars in Mesoamerica and Peru: Native American Computations of Time, pp. 1-26.

1992 "A Solution for the Number 1.5.5.0 of the Maya Venus Table", in Aveni, The Sky in Mayan Literature, pp. 207-215.

Owen, Nancy Kely

n. d. "The Dresden Cosmological Calendars", forthcoming.

Rose, Lynn E., AND RAYmond C. VAughan

1980 "Section II: The Artificial Insertion", Kronos, V: 4 (Summer, 1980), pp. 32-47.

Teeple, John E.

1926 "Maya Inscriptions: The Venus Calendar and Another Correlation", American Antbropologist, XXVIII: 2 (April, 1926), pp. 402-408.

1930 Maya Astronomy, in Contributions to American Archaeology, Volume I, Number 2. Washington: Carnegie Institution of Washington, Publication No. 403, November, 1931, "Preprinted August 1930", pp. 29-115.

Thompson, J. ERIC S.

1950 Maya Hieroglyphic Writing: Introduction. Publication 589. Washington, D.C.: Carnegie Institution of Washington.

1974 "Maya Astronomy", in The Place of Astronomy in the Ancient World, edited by F. R. Hodson. Published for the British Academy. London: Oxford University Press.

VAUGHAN, RAYMOND C.

n. d. Fortran program, V87ARC, unpublished. 